\title{
Six Sigma Quality Approach to Robust Optimization
}

\author{
Song Xiao, Yinjiang Li, Mihai Rotaru, and Jan K. Sykulski, Fellow, IEEE \\ Electronics and Computer Science, University of Southampton, Southampton SO17 1BJ, U.K.
}

In electromagnetic design, uncertainties in design variables are inevitable, thus in addition to pursuing the theoretical optimum of the objective function the evaluation of robustness of the optimum solution is also critical. Several methodologies exist to tackle robust optimization, such as worst case optimization and gradient index; this paper investigates the use of standard deviation and mean value of objective function under uncertainty of variables. A modified Kriging model with the ability of balancing exploration and exploitation is employed to facilitate the objective function prediction. Two TEAM benchmark problems are solved using different methodologies to compare the advantages and disadvantages of different robust optimization approaches.

Index Terms - Gradient index (GI), Kriging, six sigma quality (SSQ) approach, worst case optimization (WCO).

\section{INTRODUCTION}

$\mathbf{I}$ N PRACTICAL electromagnetic problems, design variables are often subject to tolerances or uncertainties; various ways of assessing performance variation under uncertain conditions have been tried, the most popular being the worst case optimization (WCO) [1]-[4], gradient index (GI) [5], [6], and six sigma quality (SSQ) [7], [8]. These methods are compared in this paper and a technique, which combines the WCO and GI with the six sigma approach is introduced and explained. The six sigma techniques for process improvement and to aid business strategies were proposed in mid 1980s and recently used in the context of quality manufacturing [7], [8]. The combined algorithm proposed here utilizes a cheap but accurate prediction provided by the modified Kriging surrogate model, which is able to balance exploration and exploitation adaptively [9], [10]. The algorithm is verified against a demanding test function and two TEAM benchmark problems.

\section{RoBUST OptIMIZATION}

In conventional optimization, finding the minimum (maximum) of the objective function is normally set as the only task while the search space if limited through constrains. When practical devices are designed, however, most parameters (design variables) are subject to uncertainties due to manufacturing tolerances, variation of material properties, and so on, and their influence on performance needs to be known. Thus, in addition to finding the theoretical optimum, its robustness may need to be assessed, often quantitatively.

\section{A. Robust Optimization}

To illustrate the importance of robustness, an example will be shown using the following test function:

$y=10-\sum_{i=1}^{n}\left[\frac{3.5}{1+\left(x_{i}-5\right)^{2}}+\frac{2.2}{1+\left(x_{i}-15\right)^{2} / 10}+\frac{1.2}{1+\left(x_{i}-25\right)^{2} / 30}\right]$

where the range $0 \leq x \leq 27$ has been considered.

The objective function $f(x)$ is to be minimized subject to constraints and uncertainties; the latter may be specified directly (e.g. as machining tolerances, say $\Delta$ ) or defined as

$$
U(x)=\left\{\xi \in R^{n} \mid x-k \sigma \leq \xi \leq x+k \sigma\right\}
$$

Manuscript received May 19, 2014; revised August 28, 2014 and September 23, 2014; accepted September 24, 2014. Date of current version April 22, 2015. Corresponding author: J. K. Sykulski (e-mail: jks@soton.ac.uk).

Color versions of one or more of the figures in this paper are available online at http://ieeexplore.ieee.org.

Digital Object Identifier 10.1109/TMAG.2014.2360435

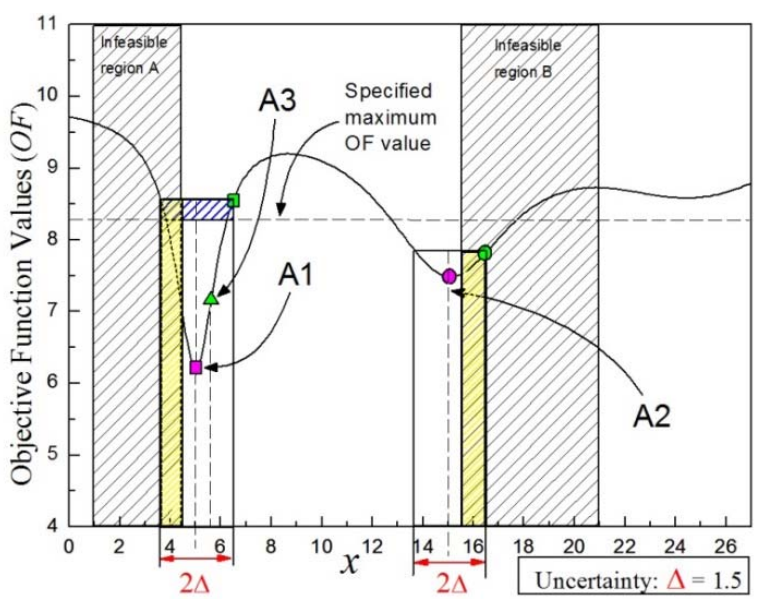

Fig. 1. Objective function and the standard deviation $(\Delta(x)=1.5)$.

where $\sigma$ is standard deviation of uncertain variables and $k$ is determined by a confidence level [5]. The single-variable $(n=1)$ version is plotted in Fig. 1 with the corresponding standard deviation for the assumed uncertainty $\Delta(x)=1.5$.

In practical cases, the design vector is often constrained, as shown in Fig. 1. Point $A 1$ is the theoretical global optimum, but after considering, the uncertainty clearly offers inferior robustness compared with $A 2$, even if the latter is only a local minimum. The infeasible regions $3.5<x<6.5$, and $15.5<x<21$ are restricted by constraints on the variable $x$. In addition, there may exist an imposed requirement for the objective function not to exceed a certain value, as depicted by the horizontal dashed line at $y=8.35$, further reducing the quality of the solution given by $A 1$. Overall, reliable ways of making a judgment about the robustness are required.

\section{B. Worst Case Optimization}

The WCO [1]-[3], [11] is a popular approach, which can predict the worst scenario considering uncertainties and constraints with respect to specific designs by performing

$$
\begin{aligned}
& \min \quad f_{w}(x) \equiv \max _{\xi \epsilon U(x)} f(\xi) \\
& \text { s.t. } \quad g_{w, i}(x)=\max _{\xi \epsilon U(x)} g(x) \leq 0, \quad i=1, \ldots, m .
\end{aligned}
$$

For a given uncertainty of a variable (or a set of variables), the worst value of the objective function in a given range (surrounding a selected point $x$ ) is used instead the original value at $x$. Thus, the worst possible performance in the vicinity of an optimum is considered directly. In addition, solutions that may push the function into unfeasible region restricted by 


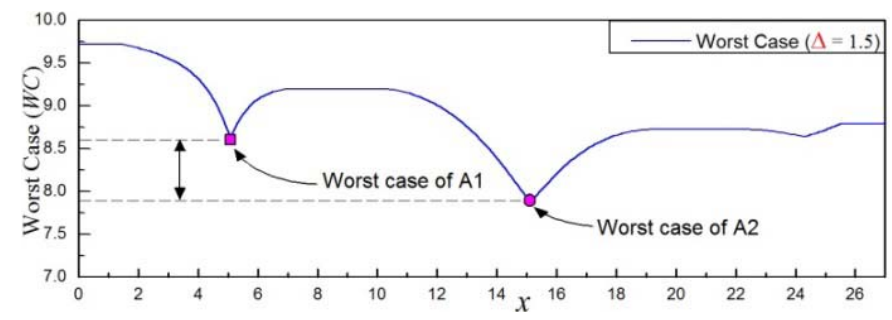

Fig. 2. Performance of the WCO method.
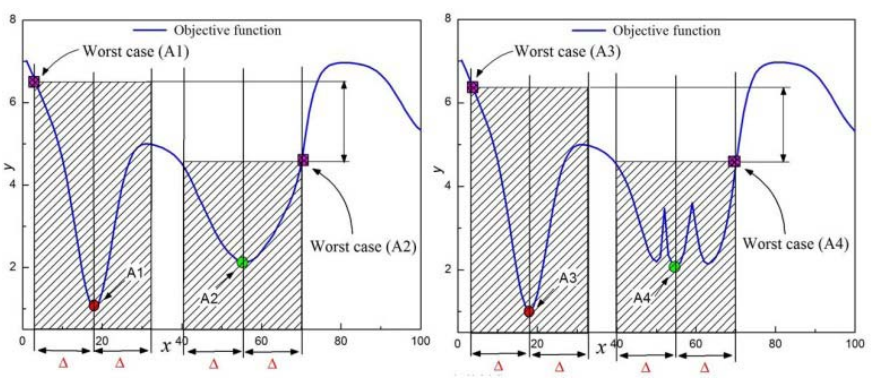

Fig. 3. Example variations of the objective function close to a local minimum.

TABLE I

COMMON FEATURES OF THE TWO FunCTIONS

\begin{tabular}{cccc}
\hline \hline $\mathrm{GM}(\mathrm{A} 1, \mathrm{~A} 3)$ & $\mathrm{LM}(\mathrm{A} 2, \mathrm{~A} 4)$ & $\mathrm{WC}(\mathrm{A} 2, \mathrm{~A} 4)$ & $\mathrm{AV}(\mathrm{A} 2, \mathrm{~A} 4)$ \\
\hline$x=18, y=1.022$ & $x=55, y=2.127$ & $x=70, y=4.478$ & 2.9556 \\
\hline \hline
\end{tabular}

constraints may be avoided. The results of the worst case (WC) analysis of the objective function (2) (of Fig. 1) are shown graphically in Fig. 2, under the uncertainty $\Delta(x)=1.5$; clearly $A 2$ offers superior performance.

Comparing only the $\mathrm{WC}$, however, means that the average variance within each range is ignored. Fig. 3 shows two functions, otherwise identical, except for the region around the points $A 2$ and $A 4$, respectively, with more details in Table I. The shape of the functions is clearly different, but all the descriptors-global minimum, local minimum, $\mathrm{WC}$, and even average value (AV) - are the same. Thus, the WCO, even with the added measure of AV, may not be reliable.

The WCO may, however, be utilized to map the probability of constraint violation. The probability of constraint violation for the function of Fig. 1, which is the measure of how likely the constraint violation may occur out of all the potential possible values in the uncertain range, is plotted in Fig. 4. $A 1$ has a slightly lower probability of $32 \%$, whereas $A 2$ has probability of $35 \%$. The WC approach could, therefore, be used to evaluate the probability of constraint violation.

\section{Gradient Index}

Another way of incorporating robustness into the mainstream optimization process is by adding the GI [5] as a second objective and formulating the problem as

$$
\begin{array}{ll}
\min & f(x) \quad x \in R^{n}\left(x_{L} \leq x \leq x_{U}\right) \\
\min & \mathrm{GI}(x)=\max _{1 \leq i \leq n}\left|\partial f(x) / \partial x_{i}\right| \\
\text { s.t. } & g_{i}(x) \leq 0, \quad i=1, \ldots, m .
\end{array}
$$

The values of the first-order gradient for points $A 1$ and $A 2$ are both close to zero. Another point, $A 3$, from Fig. 1 appears to offer a better objective function value than $A 2$, but its robustness is poor. Thus, only minimizing the first- or secondorder gradient (Fig. 5) may not offer reliable criteria.

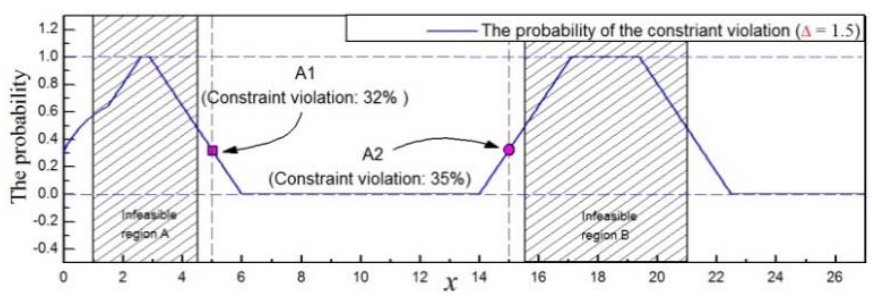

Fig. 4. Probability of constraint violation.

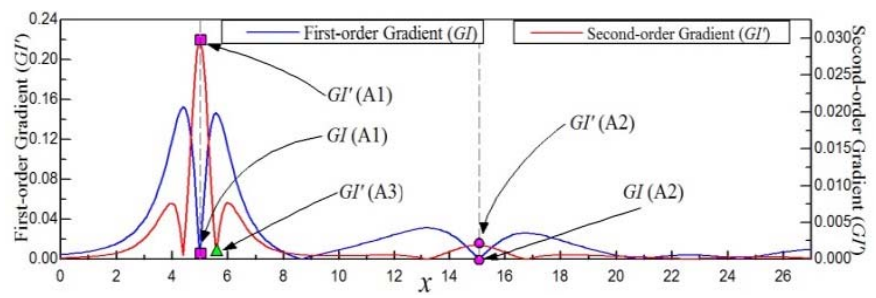

Fig. 5. First-order and second-order gradient indices of the objective function.

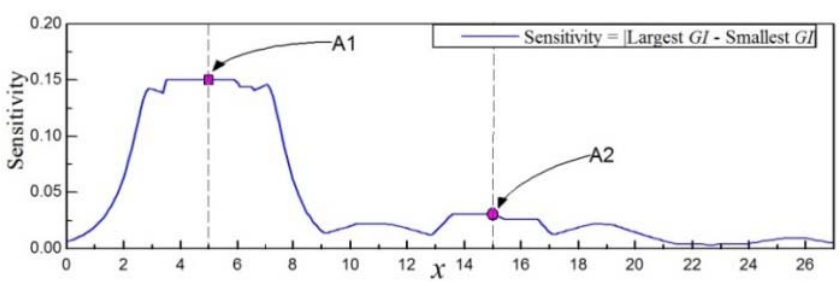

Fig. 6. Sensitivity of the objective function.

In addition, the size of the uncertainty matters. It is helpful to define the sensitivity of the gradient as the difference between the largest and the smallest value of the GI within the uncertainty range; the shape of this sensitivity carries useful information, as shown in Fig. 6. However, the assessment of average performance variation should be carried out too.

\section{SSQ Method}

To improve the quality of the assessment of robustness, as well as reduce computation times, the SSQ method is proposed to provide reliable evaluation while assessing the average performance. In addition, this now forms an inherent part of the formulation. With SSQ, the measure of dispersion is classified into six sigma (standard deviation) levels [7]; the optimization problem can be reformulated as

$$
\min \mu_{f} \text { and } \sigma_{f}
$$

where $\sigma_{f}$ is the standard deviation indicating the intensity of variation due to the uncertainty of variables and $\mu_{f}$ the mean value defining the average performance within the uncertain range. The two parameters, $\sigma_{f}$ and $\mu_{f}$, for the test function (2) are shown in Figs. 7 and 8, respectively. The trajectory of the objective function in terms of standard deviation and mean value is plotted in Fig. 9 with reference to characteristic points from Fig. 1. The standard deviation of $A 2$ at 0.0302 is less than the value for $A 1$ at 0.1499 implying better robustness.

\section{Robust Optimization EXPLOITING KRIGING}

\section{A. Kriging}

The methods discussed in Section II were combined with a Kriging-assisted surrogate model [10], [11], considering both unconstraint and constraint optimization. For the unconstraint 


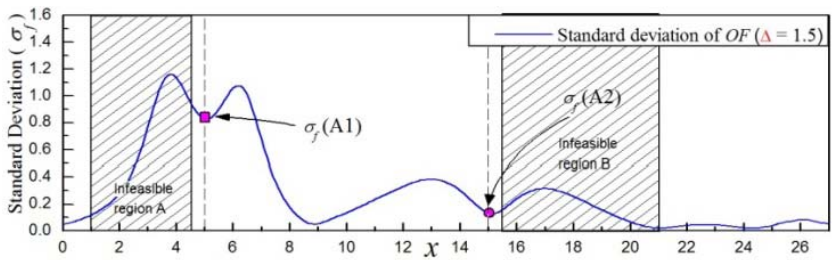

Fig. 7. Standard deviation of the objective function.

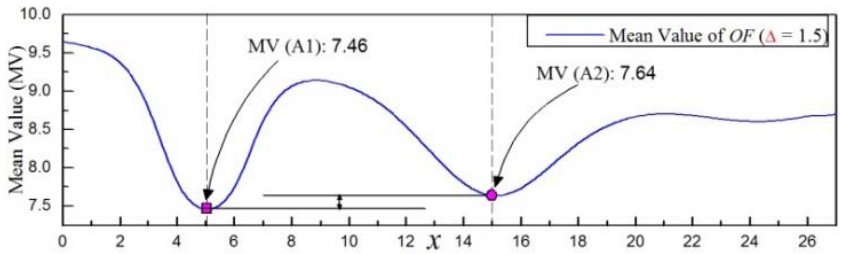

Fig. 8. Mean value of the objective function.

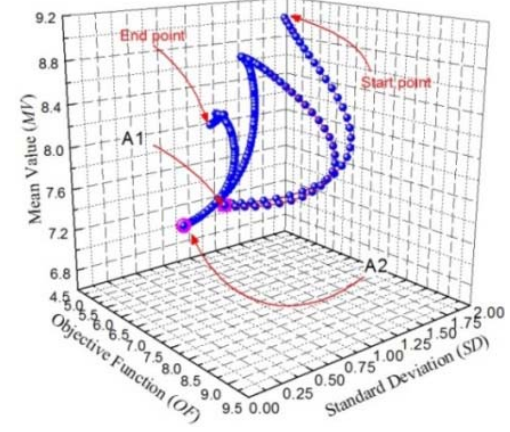

Fig. 9. Full-field solution for three objectives.

case, once the prediction of the objective function is provided by the Kriging model, the sensitivity can be evaluated using either the gradient difference or the SSQ method. The latter can also assess the average performance. The sensitivity is then used to gauge the robustness of the solution. For constraint optimization, Kriging can predict the objective function but also the constrained values. The WC method is applied to compute the probability of constraint violation and then either the GI or the six sigma algorithm is used to calculate the sensitivity to assess the robustness. The methodology will now be verified using two TEAM problems and compared with published results.

\section{B. TEAM 22 Problem}

The first example involves a multi-objective version of the TEAM 22 problem described fully in [12]. The target is an arrangement of two superconducting coils yielding the stored energy of $E_{\text {ref }}=180 \mathrm{MJ}$ while a minimal stray field $B_{\text {stray }}$ should be maintained. The objective function is defined as

$$
\mathrm{OF}=\frac{B_{\text {stray }}^{2}}{B_{\text {norm }}^{2}}+\frac{\left|E-E_{\text {ref }}\right|}{E_{\text {ref }}}
$$

where $B_{\text {norm }}=3 \mu \mathrm{T}$ and $B_{\text {stray }}^{2}=\Sigma_{i=1}^{22}\left|B_{\text {stray, } i}\right|^{2} / 22$, subject to geometrical and quench constraints. The three parameter case, which includes three geometric variables $R_{2}, H_{2}$, and $D_{2}$, while $R_{1}, H_{1}$, and $D_{1}$ are fixed, has been tried under different uncertainties, which can exist in the geometric variables or the current densities in the coils. The uncertainties have been set as $R_{2}-0.03<\xi\left(R_{2}\right)<R_{2}+0.03, R_{2}-0.042<\xi\left(h_{2}\right)<$ $R_{2}+0.042, d_{2}-0.009<\xi\left(d_{2}\right)<d_{2}+0.009$. A constraint was imposed that the superconducting coils should not violate
TABLE II

SPECIFIC SETTINGS OF THE TEAM 22 TEST

\begin{tabular}{cccc}
\hline \hline & $R_{2}(\mathrm{~m})$ & $h_{2}(\mathrm{~m})$ & $d_{2}(\mathrm{~m})$ \\
\hline Test range & {$[2.63 .4]$} & {$[0.4082 .2]$} & {$[0.10 .4]$} \\
Step size & 0.01 & 0.014 & 0.003 \\
Number of steps & 81 & 129 & 101 \\
\hline \hline
\end{tabular}

TABLE III

PERFormance COMPARISON OF Algorithms FOR TEAM 22 PROBLEM [13]

\begin{tabular}{cccclc}
\hline \hline Algorithm & $R_{2}(m)$ & $d_{2}(m)$ & $h_{2} / 2(m)$ & OF & $\begin{array}{c}\text { No of FEM } \\
\text { calls }\end{array}$ \\
\hline GA & 3.040 & 0.386 & 0.240 & 0.134 & 2400 \\
HuTS & 3.080 & 0.380 & 0.246 & 0.089 & 3821 \\
ITS & 3.100 & 0.388 & 0.240 & 0.098 & 1824 \\
SA & 3.078 & 0.390 & 0.237 & 0.098 & 5025 \\
NTS & 3.080 & 0.370 & 0.254 & 0.089 & 1800 \\
PBIL & 3.110 & 0.421 & 0.241 & 0.101 & 3278 \\
EI (Kriging) & 3.090 & 0.394 & 0.236 & 0.0875 & 211 \\
AWEI (Kriging) & 3.090 & 0.400 & 0.232 & 0.0875 & 323 \\
\hline \hline
\end{tabular}

Genetic Algorithm (GA) [14]; Tabu Search (HuTS) [15]; Improved Tabu Search (ITS) [16]; Simulated Annealing (SA) [17]; New Tabu Search (NTS) [18]; Population-based Incremental Learning (PBIL) [19]; Expected Improvement (EI), Adaptive Weighted Expected Improvement (AWEI) [13].

TABLE IV

DETAILED INFORMATION ABOUT THE

FOUR LABELED POINTS OF FIG. 10

\begin{tabular}{ccccccc}
\hline \hline Points & $R_{2}(m)$ & $h_{2} / 2(m)$ & $d_{2}(m)$ & SA & $\mathrm{C}$ & $\mathrm{P}(\mathrm{cv})$ \\
\hline$A_{1}$ & 3.04 & 0.246 & 0.382 & 6.232 & -0.4369 & $0 \%$ \\
$A_{2}$ & 3.06 & 0.232 & 0.397 & 6.654 & -1.2473 & $0 \%$ \\
$A_{3}$ & 3.08 & 0.246 & 0.376 & 6.105 & -0.5107 & $0 \%$ \\
$A_{4}$ & 3.03 & 0.281 & 0.397 & 6.347 & -1.3416 & $0 \%$ \\
\hline \hline
\end{tabular}

the quench condition, which links the value of the current density and the maximum value of magnetic flux density as

$$
g_{i(x)}=\left|J_{i}\right|+6.4 \cdot\left|B_{m, i}\right|-54.0 \leq 0, \quad i=1,2 .
$$

The initial sampling points could be selected using the Latin Hypercube [20]; we fixed them at $\left(R_{2}=2.7 \mathrm{~m}, h_{2}=0.744 \mathrm{~m}\right.$, and $\left.d_{2}=0.13 \mathrm{~m}\right),\left(R_{2}=2.9 \mathrm{~m}, h_{2}=1.304 \mathrm{~m}\right.$, and $\left.d_{2}=0.22 \mathrm{~m}\right),\left(R_{2}=3.0 \mathrm{~m}, h_{2}=1.64 \mathrm{~m}\right.$, and $\left.d_{2}=0.40 \mathrm{~m}\right)$, and $\left(R_{2}=3.3 \mathrm{~m}, h_{2}=2.088 \mathrm{~m}\right.$, and $\left.d_{2}=0.37 \mathrm{~m}\right)$ to facilitate comparisons between the Kriging model and other strategies. The initial settings are shown in Table II, while a comparison with other published results is described in Table III. The main advantage of the Kriging approach is a significant reduction of necessary FEM calls, while achieving a better value of objective function. Table IV and Fig. 10 show four points $A 1-A 4$ on the pareto front, hence good solutions.

\section{TEAM 25 Problem}

A model of a die press with an electromagnet for producing anisotropic permanent magnets is chosen as a second example [21]. The shape of the die is set up in such a way that magnetic flux density components $B_{x}$ and $B_{y}$ should be the same and equal to $0.35 \cos (\theta) \mathrm{T}$ along a circle line in 10 measurement points for $0^{\circ}<\theta<45^{\circ}$ and $r_{0}=0.01175 \mathrm{~m}$. The problem has four design parameters $R_{1}, L_{2}, L_{3}$, and $L_{4}$ specified in Table V.

The objective function is evaluated at specific points as

$\mathrm{OF}=\sum_{i=1}^{10}\left(\left(B_{x i, \text { calc }}-B_{x i, \text { requ }}\right)^{2}+\left(B_{y i, \text { calc }}-B_{y i, \text { requ }}\right)^{2}\right)$ 

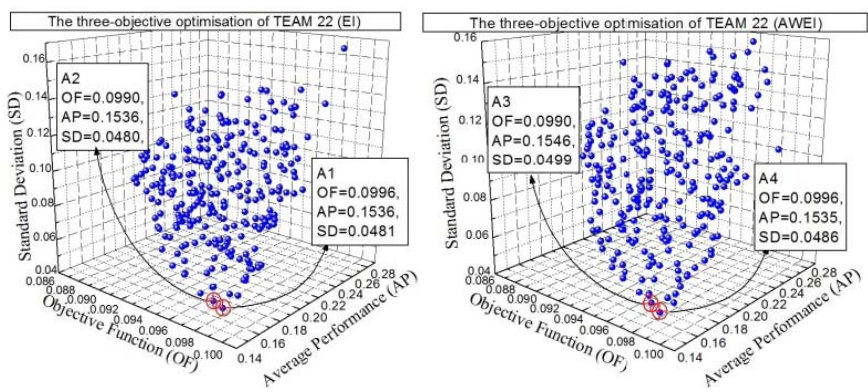

Fig. 10. TEAM 22 results. Left: Kriging with EI. Right: Kriging with AWEI (prediction of objective function, average performance and standard deviation). More information about $A 1$ to $A 4$ in Table IV (SA: sensitivity assessment; C: constraint value, $\mathrm{P}(\mathrm{cv})$ : probability of constraint violation).

TABLE V

SPECIFIC DEFINITION OF TEAM 25

\begin{tabular}{|c|c|c|c|c|}
\hline \multirow[t]{2}{*}{ Parameter } & \multicolumn{2}{|c|}{ "Test Range $(\mathrm{mm})$} & \multirow{2}{*}{$\begin{array}{c}\text { Step Size } \\
(\mathrm{mm})\end{array}$} & \multirow[t]{2}{*}{ No of Steps } \\
\hline & Min & $\operatorname{Max}$ & & \\
\hline$R_{1}$ & 5 & 9.4 & 0.1 & 45 \\
\hline$L_{2}$ & 12.6 & 18 & 0.1 & 55 \\
\hline$L_{3}$ & 14 & 45 & 1 & 32 \\
\hline$L_{4}$ & 4 & 19 & 0.5 & 31 \\
\hline
\end{tabular}

TABLE VI

PERFormance COMPARISON OF Algorithms

FOR TEAM 25 PROBLEM

\begin{tabular}{ccccccc}
\hline \hline Algorithm & $\begin{array}{c}O F \\
\left(10^{-4} \mathrm{~T}^{2}\right)\end{array}$ & $\begin{array}{c}R_{1} \\
(\mathrm{~mm})\end{array}$ & $\begin{array}{c}L_{2} \\
(\mathrm{~mm})\end{array}$ & $\begin{array}{c}L_{3} \\
(\mathrm{~mm})\end{array}$ & $\begin{array}{c}L_{4} \\
(\mathrm{~mm})\end{array}$ & $\begin{array}{c}\text { No of } \\
\text { FEM calls }\end{array}$ \\
\hline GA & 2.6861 & 7.2996 & 14.174 & 14.001 & 14.326 & 3421 \\
SA & 1.6223 & 7.2252 & 14.322 & 14.110 & 14.306 & 2145 \\
HuTS & 0.5009 & 7.3780 & 14.613 & 14.371 & 14.204 & 1580 \\
UTS & 1.0501 & 7.5487 & 14.908 & 14.506 & 14.416 & 931 \\
NTS & 0.6482 & 7.4337 & 14.732 & 14.428 & 14.237 & 575 \\
EI & 0.4527 & 7.2 & 14.1 & 14 & 14.5 & 265 \\
AWEI & 0.4125 & 7.2 & 14 & 14 & 14.5 & 214 \\
\hline \hline
\end{tabular}

Universal tabu search (UTS) [20], otherwise as in Table III.
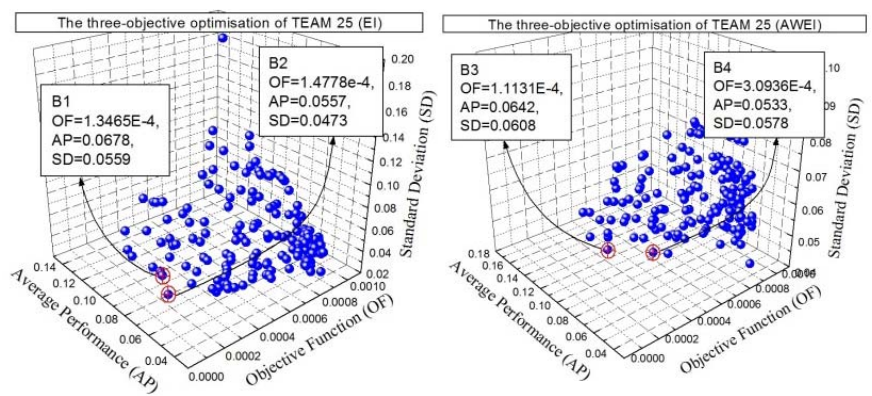

Fig. 11. TEAM 25 results. Left: Kriging with EI. Right: Kriging with AWEI (prediction of objective function, average performance, and standard deviation).

where calc means calculated and requ required. There are three initial sampling points, which are chosen randomly.

Comparison with other published methods in Table VI once again shows Kriging with EI and/or adaptive weighted expected improvement (AWEI) performing much better in terms of reducing the number of required FEM calls. The sampling points $B 1-B 4$ in Fig. 11 are all pareto optimal.

\section{CONCLUSION}

The SSQ approach, supplemented by the WC criteria and GI method and aided by Kriging surrogate modeling for efficient prediction of the objective function, has been found to outperform other methods in assessing the robustness of the different optimal solutions, while also providing additional useful information. In such assessments, there are no definite criteria for deciding which design is the best, but the proposed methodology has been shown to offer the most comprehensive treatment and results facilitating the decision making process.

\section{REFERENCES}

[1] A. Ambrisi, A. Formisano, and R. Martone, "Tolerance analysis of NMR magnets," IEEE Trans. Magn., vol. 46, no. 8, pp. 2747-2750, Aug. 2010.

[2] G. Steiner, A. Weber, and C. Magele, "Managing uncertainties in electromagnetic design problems with robust optimization," IEEE Trans. Magn., vol. 40, no. 2, pp. 1094-1099, Mar. 2004.

[3] G. L. Soares, R. Adriano, C. A. Maia, L. Jaulin, and J. A. Vasconcelos, "Robust multi-objective TEAM 22 problem: A case study of uncertainties in design optimization," IEEE Trans. Magn., vol. 45, no. 3, pp. 1028-1031, Mar. 2009.

[4] P. Alotto, C. Magele, W. Renhart, A. Weber, and G. Steiner, "Robust target functions in electromagnetic design," COMPEL, Int. J. Comput. Math. Elect. Electron. Eng., vol. 22, no. 3, pp. 549-560, 2003.

[5] Z. Ren, M.-T. Pham, M. Song, D.-H. Kim, and C. S. Koh, "A robust global optimization algorithm of electromagnetic devices utilizing gradient index and multi-objective optimization method," IEEE Trans. Magn., vol. 47, no. 5, pp. 1254-1257, May 2011.

[6] Z. Ren, M.-T. Pham, and C. S. Koh, "Robust global optimization of electromagnetic devices with uncertain design parameters: Comparison of the worst case optimization methods and multiobjective optimization approach using gradient index," IEEE Trans. Magn., vol. 49, no. 2, pp. 851-859, Feb. 2013.

[7] K. Shimoyanama, A. Oyama, and K. Fujii, "A new efficient and useful robust optimization approach-Design for multi-objective six sigma," in Proc. IEEE Congr. Evol. Comput., vol. 1. Sep. 2005, pp. 950-957.

[8] G. Lei, J. G. Zhu, Y. G. Guo, J. F. Hu, W. Xu, and K. R. Shao, "Robust design optimization of PM-SMC motors for six sigma quality manufacturing," IEEE Trans. Magn., vol. 49, no. 7, pp. 3953-3956, Jul. 2013.

[9] S. Xiao, M. Rotaru, and J. K. Sykulski, "Considerations of uncertainty in robust optimization of electromagnetic devices," in Proc. ISEF, Ohrid, Macedonia, 2013

[10] S. Xiao, M. Rotaru, and J. K. Sykulski, "Robust global optimization of electromagnetic designs utilizing gradient indices and kriging," in Proc. 19th Int. Conf. COMPUMAG, Budapest, Hungary, Jun./Jul. 2013, paper PC4-6.

[11] L. Egiziano, P. Lamberti, G. Spagnuolo, and V. Tucci, "Robust design of electromagnetic systems based on interval Taylor extension applied to a multiquadric performance function," IEEE Trans. Magn., vol. 44, no. 6, pp. 1134-1137, Jun. 2008.

[12] SMES Optimization Benchmark: TEAM Workshop Problem 22. [Online] Available: http://www.compumag.org/jsite/images/stories/TEAM/ problem22.pdf, accessed Feb. 10, 2015.

[13] S. Xiao, M. Rotaru, and J. K. Sykulski, "Adaptive weighted expected improvement with rewards approach in kriging assisted electromagnetic design," IEEE Trans. Magn., vol. 49, no. 5, pp. 2057-2060, May 2013.

[14] J. H. Holland, Adaptation in Natural and Artificial Systems: An Introductory Analysis with Applications to Biology, Control, and Artificial Intelligence. Cambridge, MA, USA: MIT Press, 1975.

[15] N. Hu, "Tabu search method with random moves for globally optimal design," Int. J. Numer. Methods Eng., vol. 35, no. 5, pp. 1055-1070, 1992.

[16] S. L. Ho, S. Yang, G. Ni, and H. C. Wong, "An improved Tabu search for the global optimizations of electromagnetic devices," IEEE Trans. Magn., vol. 37, no. 5, pp. 3570-3574, Sep. 2001.

[17] S. Kirkpatrick, C. D. Gelatt, Jr., and M. P. Vecchi, "Optimization by simulated annealing," Science, vol. 220, no. 4598, pp. 671-680, 1983.

[18] O. Hajji, S. Brisset, and P. Brochet, "A new Tabu search method for optimization with continuous parameters," IEEE Trans. Magn., vol. 40, no. 2, pp. 1184-1187, Mar. 2004.

[19] S. Yang, Y. Bai, G. Zhang, and L. Wu, "An improved populationbased incremental learning method for inverse problems," in Proc. World Autom. Congr., Sep./Oct. 2008, pp. 1-4.

[20] T. J. Santner, B. J. Williams, and W. I. Notz, The Design and Analysis of Computer Experiments. New York, NY, USA: Springer-Verlag, 2003.

[21] N. Takahashi, "Optimization of die press model," in Proc. TEAM Workshop 6th Round, Okayama, Japan, Mar. 1996, pp. 20-21. 